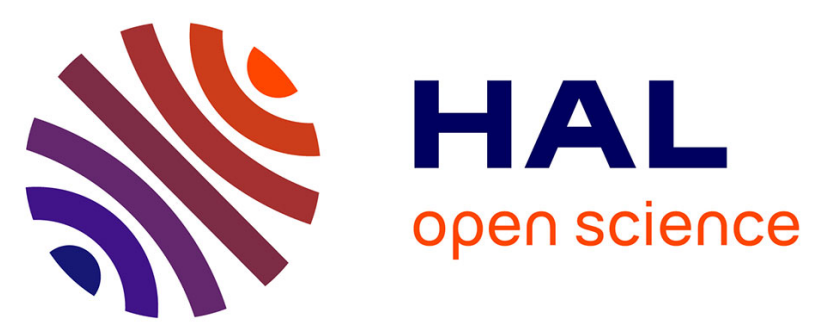

\title{
Wavelength stabilization and control of the emission of pulsed dye lasers by means of a multibeam Fizeau interferometer
}

C. Cahen, Jean-Pierre Jegou, Jacques Pelon, P. Gildwarg, Jacques Porteneuve

\section{- To cite this version:}

C. Cahen, Jean-Pierre Jegou, Jacques Pelon, P. Gildwarg, Jacques Porteneuve. Wavelength stabilization and control of the emission of pulsed dye lasers by means of a multibeam Fizeau interferometer. Revue de Physique Appliquée, 1981, 16 (6), pp.353-358. 10.1051/rphysap:01981001606035300 . jpa00244927

\section{HAL Id: jpa-00244927 https://hal.science/jpa-00244927}

Submitted on 1 Jan 1981

HAL is a multi-disciplinary open access archive for the deposit and dissemination of scientific research documents, whether they are published or not. The documents may come from teaching and research institutions in France or abroad, or from public or private research centers.
L'archive ouverte pluridisciplinaire HAL, est destinée au dépôt et à la diffusion de documents scientifiques de niveau recherche, publiés ou non, émanant des établissements d'enseignement et de recherche français ou étrangers, des laboratoires publics ou privés. 


\title{
Wavelength stabilization and control of the emission of pulsed dye lasers by means of a multibeam Fizeau interferometer
}

\author{
C. Cahen, J. P. Jegou, J. Pelon, P. Gildwarg and J. Porteneuve \\ Service d'Aéronomie du CNRS, BP 3, 91370 Verrières le Buisson, France.
}

(Reçu le 20 novembre 1980, révisé le 18 février 1981, accepté le 20 février 1981)

\begin{abstract}
Résumé. - Nous avons construit et testé au cours d'expériences lidar de routine un système interférométrique qui stabilise et contrôle la longueur d'onde d'émission de lasers pulsés à colorant délivrant des impulsions de quelques nanosecondes ou de quelques microsecondes. Ce système permet une commutation rapide, toutes les secondes, entre des longueurs d'onde prédéterminées. La résolution effective de l'appareil est de $4 \times 10^{6}$.
\end{abstract}

\begin{abstract}
An interferometric system to stabilize and control the emission wavelength of pulsed dye lasers with nanosecond or microsecond pulse duration has been constructed and tested during routine lidar field experiments. Switching between predetermined wavelengths can also be performed within short time intervals (1 s). The effective resolution of the system is $4 \times 10^{6}$.
\end{abstract}

1. Introduction. - The determination by laser soundings (lidar technique) of physical parameters and minor constituent concentrations in the atmosphere is a continuously expending experimental field [1]. The usual spectral requirements on linewidth and line positioning accuracy for atomic or molecular spectroscopy are more difficult to fulfil due to the use of high energy pulsed lasers with narrow line emission necessary for remote measurements. Narrow line emission and high output laser energy are some contradictory requirements which can be partially overcome using injection locking or amplifying techniques or a combination of them $[2,3]$. Measurements using the differential absorption lidar technique (DIAL) [4] will also need a rapid switching between two or more predetermined wavelengths corresponding to different values of the absorption cross section for the species under study. Furthermore, the use of lidar systems for ground based continuous monitoring or for airborne experiments requires a near complete automatization of the experimental system. We have thus designed and constructed an operational device for wavelength control and stabilization based on an interferometric analysis of the laser line by means of a multibeam Fizeau interferometer associated with a multichannel electronic control system.

The choice of the optical system will be referenced in the paragraph 2 ; a description of the experimental set-up will be given in paragraph 3 and the electronic processing and data acquisition is described in paragraph 4 . The overall performances of the system and its application to specific operational lidar measurements are the subject of paragraphs 5 and 6 .

2. Optical analyser. - 2.1 THE CHOICE OF AN INTERFEROMETER. - The choice of the optical element used to perform the spectral analysis of the laser line has to take into account the specific requirements of the lidar experiments. A determining parameter is then the ratio between the laser linewidth and the stability of the emission wavelength. Whereas the linewidth itself for various studies may vary by at least 2 orders of magnitude depending on the nature of the atomic or molecular transitions studied (electronic, vibration, rotation bands, ...), the ratio between the linewidth and the line positioning is shown to be conservative and of the order of 10 [5]. Besides this requirement, the wavelength excursion range necessary to obtain a significant change of the absorption by the constituent under study is generally no larger than 3 to 5 times the absorption linewidth. Thus an optical analysis system with a ratio of 100 between total spectral range and its apparatus function will be well adapted to the measurement, provided that the range itself could be varied for each specific experiment by changing the dispersive element. All these considerations led us to the choice of interferometric devices; among the interferometers, the multibeam Fizeau 
interferometer show specific features well adapted to a suitable optical stabilization scheme :

- an interference pattern where the fringes are lines matching the detector geometry of a photodiode array [6],

- a linear phase variation on the line of greatest slope simplifying the servo-locking process,

- a non-critical positioning of the detector mount in the interference field.

These characteristics are analysed in the next section.

2.2 Multibeam Fizeau interferometer. - The theory has been developed by Brossel [7], Born and Wolf [8] and only the main results will be summarized here. In the case of a normal incidence and if one neglects the refraction effects, the difference in the optical paths between the $p$ th wave $\left(\Sigma_{p}\right)$ and the directly transmitted wave $\left(\Sigma_{0}\right)$ at a point $\mathrm{M}\left(X_{0}, Y_{0}\right)($ Fig. 1)

$$
\delta p=X_{0}(\cos 2 \varphi-1)+Y_{0} \sin 2 \varphi
$$

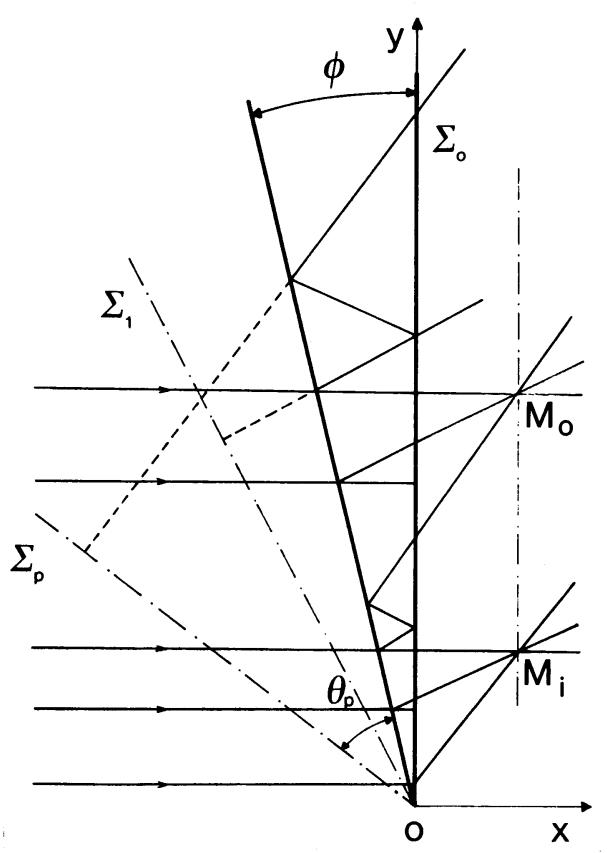

Fig. 1. - Ray-tracing of the representation of the interference pattern.

where $Y_{0}=e / \operatorname{tg} \varphi, \varphi$ is the wedge angle and $e$ the interferometer thickness assumed constant over the interference field. For high reflectivity plates $(R>0.9)$ the largest value of $p$ which gives a significant contribution to the interference pattern and consequently has to be retained is of the order of $10^{2}$. Then neglecting the variations of $\delta p$ along the $X$ axis and using a serial expansion in powers of $\varphi$ of the third order, one obtains a simplified expression of $\delta p$

$$
\delta p=2 p e-\frac{4}{3} p^{3} e \varphi^{2}
$$

This expression separates into two terms :

- the first one, 2 pe, leads to a spectral intensity distribution similar to the one obtained for the plane parallel Fabry-Perot etalon (F.P.) so that, by analogy, the interferometer resolving power, finesse and free spectral range (F.S.R.) can be defined [8] ;

- the second term, $4 / 3 p^{3} e \varphi^{2}$, introduces a distortion into the spectral intensity distribution. The magnitude of this distortion is given by the comparison

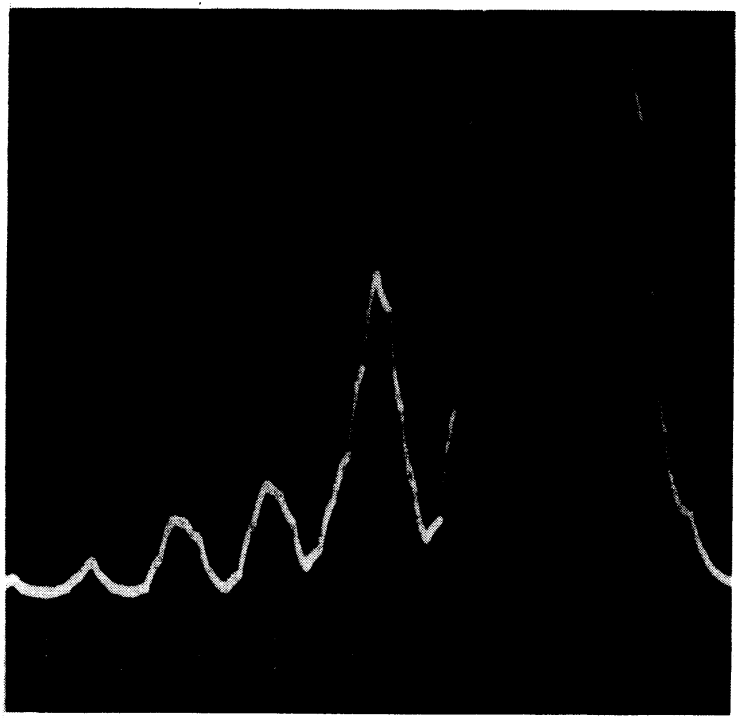

Fig. 2. - Experimental apparatus function of a Fizeau interferometer ( $e=4.5 \mathrm{~mm}, R=0.95$ ) as determined using a single longitudinal mode stabilized $\mathrm{CW}$ He-Ne laser as a source. One division corresponds to : $0.6 \mathrm{pm}$ or a phase difference of $10^{-2} \times 2 \pi$ for the He-Ne wavelength. The ordinate unit is arbitrary in order to point out the boundings and the distortion of the spectral distribution intensity.

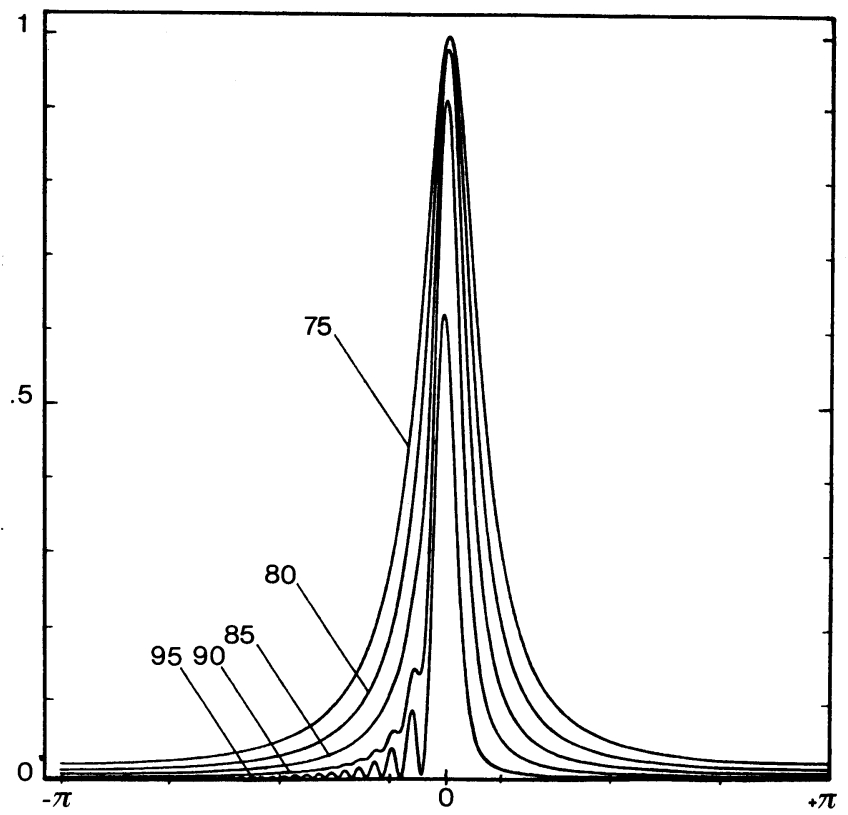

Fig. 3. - Computed apparatus function for the following parameters : $e=4.5 \mathrm{~mm}, \theta \sim 0$ and different values of the reflectivity coefficient $R$. The ordinate is normalized to the unity for $R=0 \%$. Comparison with figure 2 results are in good agreement. 
between the magnitude of this 2 nd term and $\lambda$ since a change of $\lambda$ in the optical path corresponds to a $2 \pi$ change in the phase function. If $4 / 3 p^{3} / e \varphi^{2} \ll \lambda$, the distortion is negligible. However for our usual experimental conditions in the visible region $\left(\lambda=632.8 \mathrm{~nm}, e=4 \mathrm{mn}\right.$ and $\varphi=10^{\prime \prime}$ (see 3.1)), one finds a value of $4 p^{3} e \varphi^{2} / 3 \lambda$ equal to 20 . The resulting pattern distortion is then apparent in the figure 2 which displays the experimental apparatus function recorded with a stabilized C.W. He-Ne laser. This experimental result agrees with the apparatus. function computed (Fig. 3) for different values of the parameters by adding up the complex amplitudes at the point $M$ in the interference field (see Fig. 1).

3. Experimental set-up. - 3.1 Specifications. To take into account the dynamical range of the system as previously defined (see 2.1) a finesse value of $\sim 100$ is required corresponding to a reflexion coefficient of the plates $R=0.98$ and a flatness of $\lambda / 200$. A minimal transverse dimension $\Delta Y$ along the $Y$ axis (see Fig. 1) may be determined to satisfy the complete geometric build up of a fringe. From the figure 1, $\Delta Y$ as related to a one fringe build up is expressed by :

$$
\Delta Y=2 p^{2} e \varphi .
$$

By equating this value to $\alpha / 2$ where $\alpha$ is the interfringe interval as given by :

$$
\alpha=1 / 2 \frac{\lambda}{\varphi}
$$

an optimum value of the wedge angle $\varphi\left(\sim 10^{\prime \prime}\right)$ is obtained for the given value of the finesse. Then, the minimum value of the transverse dimension of the detector and coated plate diameter is calculated to be $12 \mathrm{~mm}$. To obtain the highest value of the resolving power the incident beam has to be collimated as well as possible : the source extension and the focal length of the collimating lens are defined according to the criterion of Dufour and Picca [8] which leads to a typical value of the angular aperture $\sim 10^{-3} \mathrm{rad}$. To ensure a high thermal stability of the interferometer, the two plates are optically contacted with a low expansion glass-ceramic. The usefull coated aperture has a diameter of $16 \mathrm{~mm}$. Furthermore, to monitor the long term variation of the fringes position with respect to the detector array, a stabilized single longitudinal mode $\mathrm{He}-\mathrm{Ne}$ laser is used and the wavelength of the pulsed laser line is then retriewed with respect to the He-Ne identified diode cell. For absolute wavelength measurement, the system can be calibrated using a monochromatic source (atomic or molecular reference) of known wavelength within the same order of interference of the laser wavelength; the position of the He-Ne beam in terms of diode cell can then be calculated and expressed as an absolute value.

3.2 EXPERIMENTAL SYSTEM. - The experimental set-up is described in the figure 4. The semi-transparent thin plate (1) and the divergent lens (2) are used to mix the laser beam and the reference beam (e.g. He-Ne laser beam). The microscope objective (3) focuses the two beams on the pinhole (4). An achromatic lens (5)

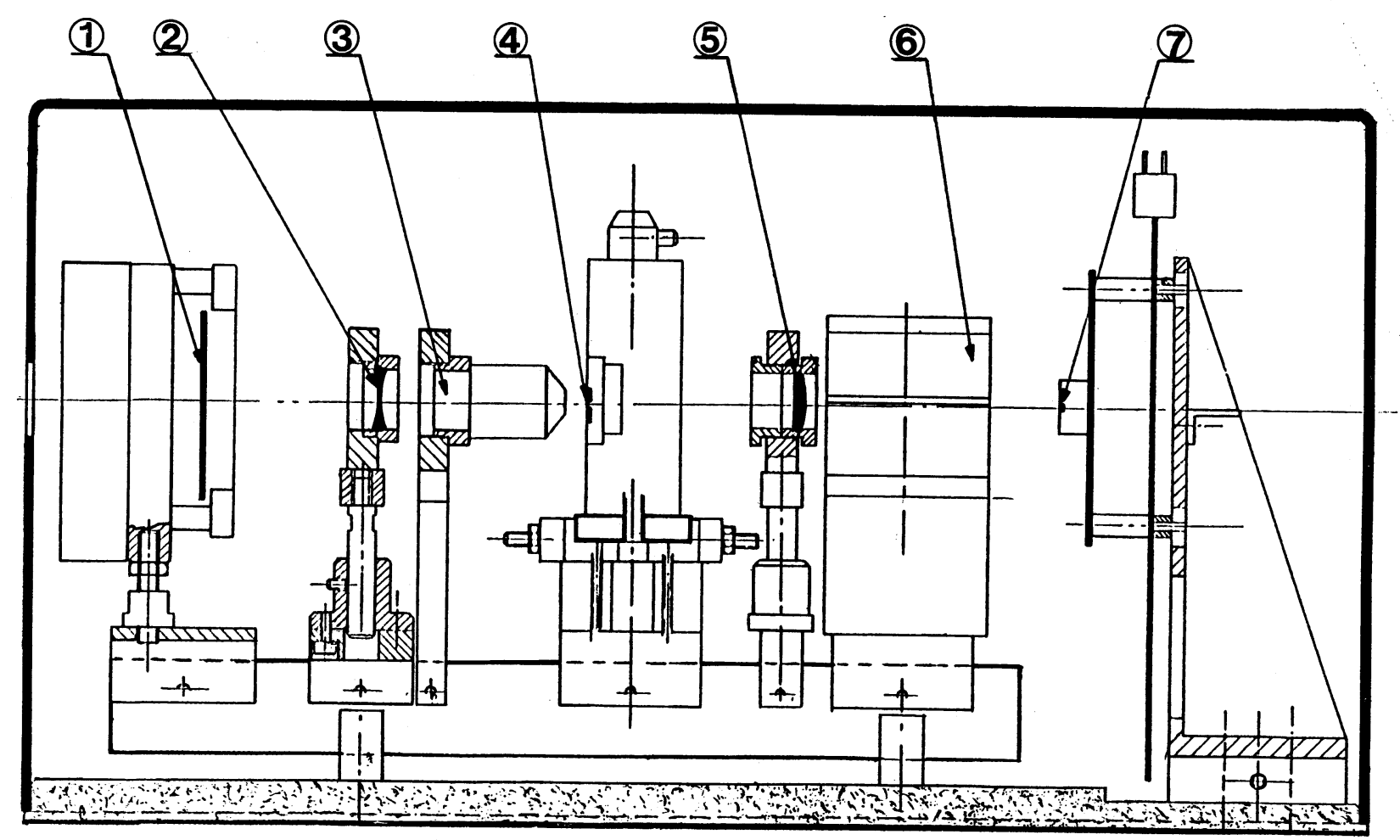

Fig. 4. - Optical set-up of the Fizeau interferometer analyser : (1) semi-transparent thin plate, (2) divergent lens $(f=-30 \mathrm{~mm}, \varnothing 18 \mathrm{~mm})$, $(3)$ microscope objective $(f=16 \mathrm{~mm}, \varnothing 8 \mathrm{~mm})$, (4) pinhole $(20 \mu \mathrm{m})$, (5) achromatic lens $(f=60 \mathrm{~mm}, \varnothing 18 \mathrm{~mm})$, (6) interferometer,
detector. 
collimates the beam onto the interferometer (6). The detector (7) is a self-scanned photodiode array from Reticon [9] with 512 bar-shaped cells with a width of $25 \mu \mathrm{m}$. This device is operated in the charge- storage mode leading to a high sensitivity [10]. The total length of the optical beach is $380 \mathrm{~mm}$. The whole system is thus very compact and mechanically stable. A photograph of the system is shown in the figure 5 .

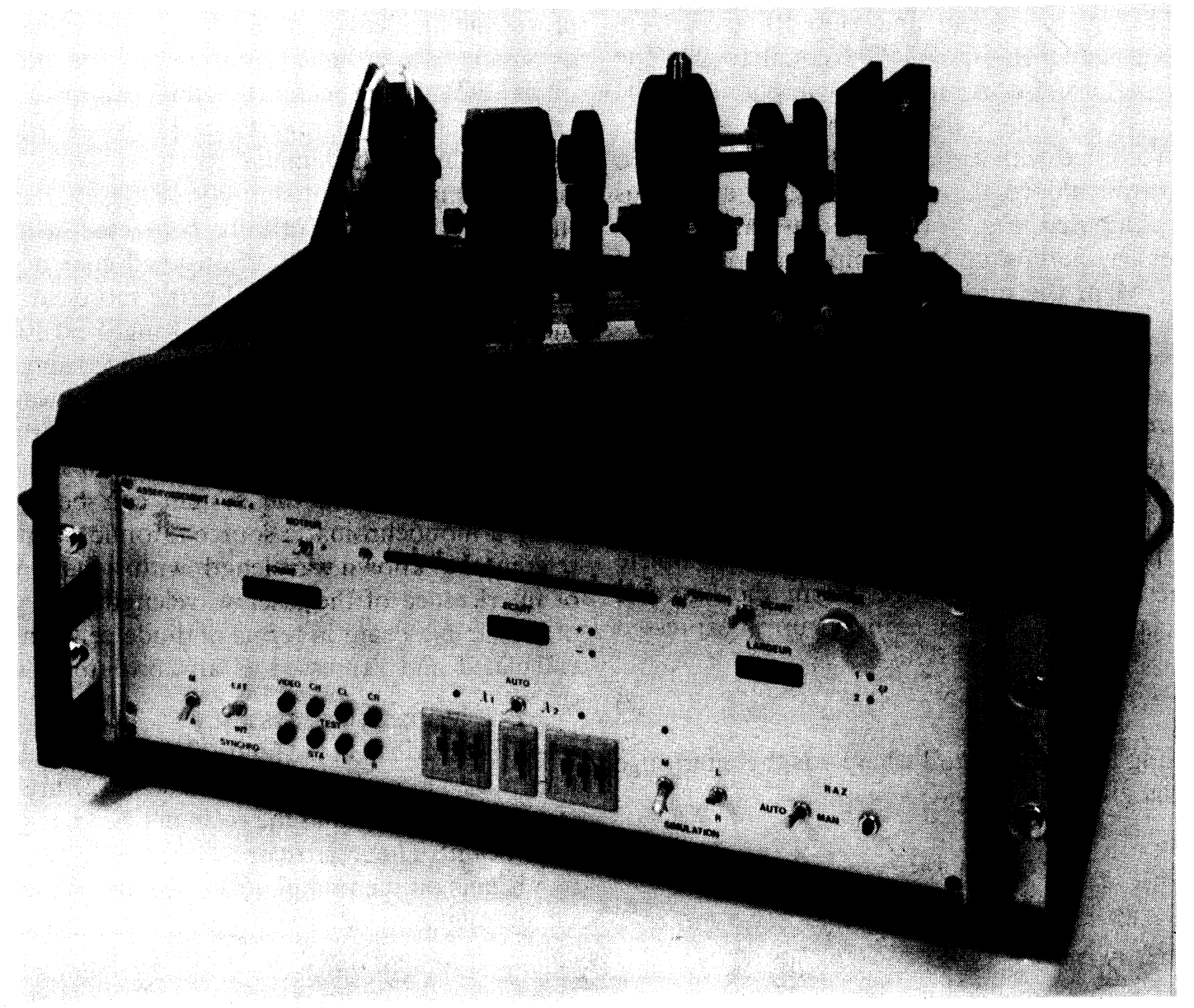

Fig. 5. - Photograph of the optical set-up, showing its compactness and its associated electronic package in a ground-based configuration.

4. Electronic processing. - The typical electronic processing includes the following steps (Fig. 6). First the reference beam is blocked and the electronic functions are reset to their initial values. The laser is triggered and part of the beam is sent onto the interferometric device. The photodiode array gives an opto-electronic readout. The output is then digitized and transferred into a buffer memory; this memory can be either a $512 \times 8$ bits memory as part of the device itself or the central memory of any other processor. The array scanning frequency is limited by the transfer speed to the buffer memory. The scanning time for the 512 cells is equal to $4 \mathrm{~ms}$. The relative laser energy and the line center position are computed from the stored data. The reference beam is then unblocked and the position of the illuminated cell is processed by a video scan (an electronic simulation of this second scan can also be preprogrammed if no laser reference is used). The difference in number of cells between the reference line center and the laser line center is then measured and converted into an error signal applied to a stepping motor tuning a laser oscillator element (F.P. or grating) which controls the emission wavelength. The loop gain applied on the error signal is adjusted with respect to the transfer function of this wavelength selector ( $\lambda$ as a function of the incidence angle).

For dual wavelength measurements (e.g. differential absorption) the wavelength of the laser emission has to be switched between two predetermined values. Thèse values in terms of diode cell position are stored in the logical memory. A new logical step is added in the electronic processing : the difference between the two references is converted into a presetted switching signal which can be applied to the stepping motor. Its duration is imposed by the value of the shift between the references and by the speed of the stepping motor. When it occurs, the control sequence onto a reference is unlocked until the laser line lies within the error range (a tenth of the laser linewidth, see table I) of the new reference. The time interval between two automatic switchings is preprogrammed with 


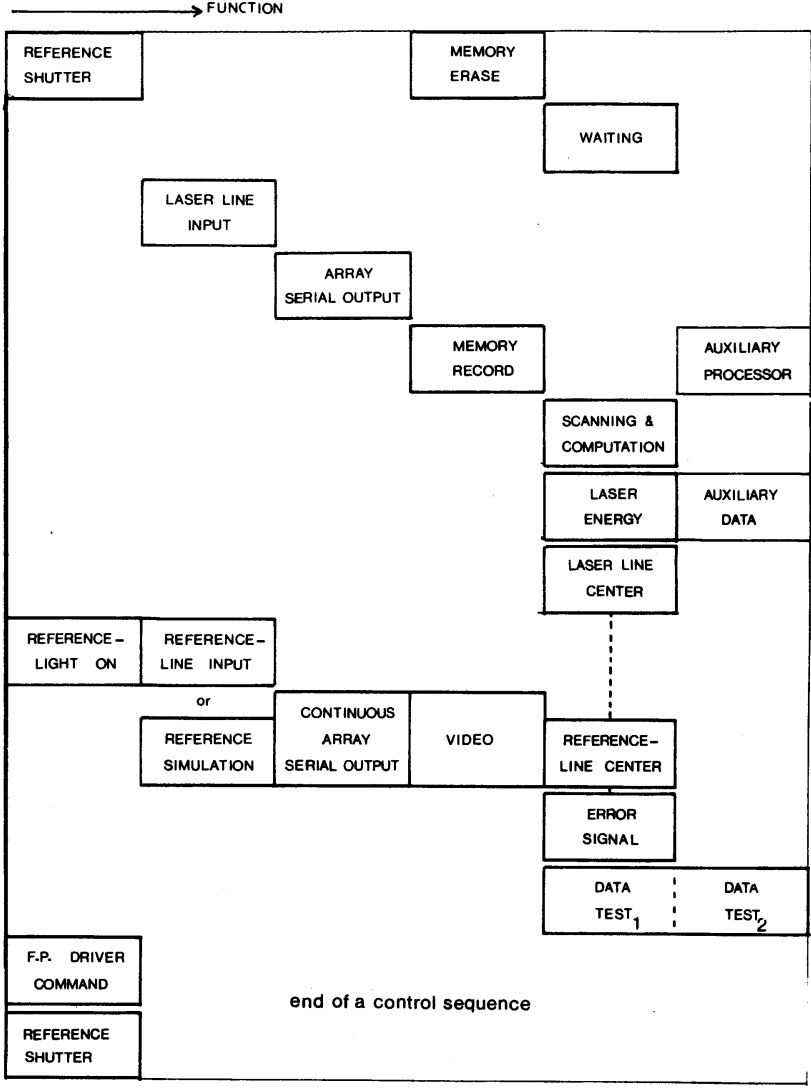

Fig. 6. - Flow diagram showing the electronic functions of the wavelength stabilization scheme. respect to the characteristics required by the atmospheric measurements (see chap. 6).

5. Characteristics. - The F.S.R. of the Fizeau interferometer is adjusted to cover the $3 / 5$ of the transverse dimension of the photodiode array so that the resolution of the system is not limited by the number of diodes per unit length; 300 diodes are available within a F.S.R. whereas the optical finesse is 80. Thus, and taking into account the linearity of the optical and electronic systems, the fluctuation of the laser line on a shot to shot basis is of the order of the interferometer resolution and constant upon the F.S.R.

Whereas the apparatus function of the Fizeau interferometer is asymmetric, the value of the laser linewidth can also be determined by using the video signal delivered by the electronic system. The charge storage operation mode (see 3.2) and the inclusion of a buffer memory (see 4) made possible the use of any type of low frequency oscilloscope to record the spectra of short laser pulses even in the nanosecond time duration range. The overall electronic processing time is less than $10 \mathrm{~ms}$ so that pulsed lasers with a $100 \mathrm{~Hz}$ repetition rate can be stabilized and controlled.

As pointed out in 3.1 absolute wavelength calibrations might require the use of incoherent standard sources. To increase the light flux on the detector in these measurements one can take advantage of the

Table I.

\begin{tabular}{|c|c|c|c|c|c|c|}
\hline $\begin{array}{l}\text { Atmospheric } \\
\text { species }\end{array}$ & $\begin{array}{c}\text { Energy } \\
\mathrm{mJ}\end{array}$ & $\begin{array}{c}\text { Repetition } \\
\text { rate } \\
\mathrm{Hz}\end{array}$ & $\begin{array}{c}\text { Laser } \\
\text { linewidth } \\
\text { pm }\end{array}$ & $\begin{array}{l}\text { Fizeau } \\
\text { thickness } \\
\text { mm }\end{array}$ & $\begin{array}{c}\text { Wavelength } \\
\text { shift } \\
\text { pm }\end{array}$ & $\begin{array}{c}\text { Switching } \\
\text { rate } \\
\mathrm{Hz}\end{array}$ \\
\hline- & - & - & - & - & - & - \\
\hline $\begin{array}{l}\text { Water vapor } \\
\text { (723 nm) }\end{array}$ & 60 & 10 & 2 & 9 & 25 & 0.1 \\
\hline $\begin{array}{l}\text { Lithium isotopes } \\
\quad(671 \mathrm{~nm})\end{array}$ & 800 & 2 & 6 & 4.5 & $\begin{array}{l}15 \\
30\end{array}$ & $10^{-2}$ \\
\hline
\end{tabular}

zero curvature of the transverse optical field to insert a cylindrical lens in the front of the array or to use larger size diode cells without resolution loss.

6. Applications and performances. - This device is presently used in various field experiments at the Haute-Provence Observatory lidar facility. These include DIAL measurements involving both electronic transitions (U.V. wavelength range) [11] and vibrationrotation bands (near infrared) and atomic resonance studies in the high atmosphere [12].

One device monitors the wavelength of a flashlamp pumped dye laser operating the resonance excitation of the lithium isotopes (Fig. 7). It tunes the position of the thickest intra-cavity F.P. to stabilize the wavelength onto a reference.

Using a $4.5 \mathrm{~mm}$ thick interferometer with a finesse of 80 , the microsecond pulsed emission at $670.8 \mathrm{~nm}$ with a linewidth of $6 \mathrm{pm}$ has been stabilized during an 8 hours long experiment with a relative accuracy of $0.5 \mathrm{pm}$ corresponding to a resolution of $1 \times 10^{6}$. An illustration of the wavelength stabilization, on a shot to shot basis, is presented in figure 8 . In figure $8 a$ we represent the error signal versus time, whereas in figure $8 b$ we show the angular evolution of the tuning element (F.P.) of the laser cavity. In a similar way in the nanosecond pulse duration range, a Nd Yag pumped dye laser is stabilized by one device using a $9 \mathrm{~mm}$ thick interferometer with a relative accuracy of $0.15 \mathrm{pm}$. The emission is centered at $723.2 \mathrm{~nm}$, the laser linewidth is $2 \mathrm{pm}$ leading to a resolution of $4 \times 10^{6}$. A switching between two wavelengths is performed every $10 \mathrm{~s}$ in a time shorter than $1 \mathrm{~s}$. Taking advantage of the computer control (PDP 11/34) of the 


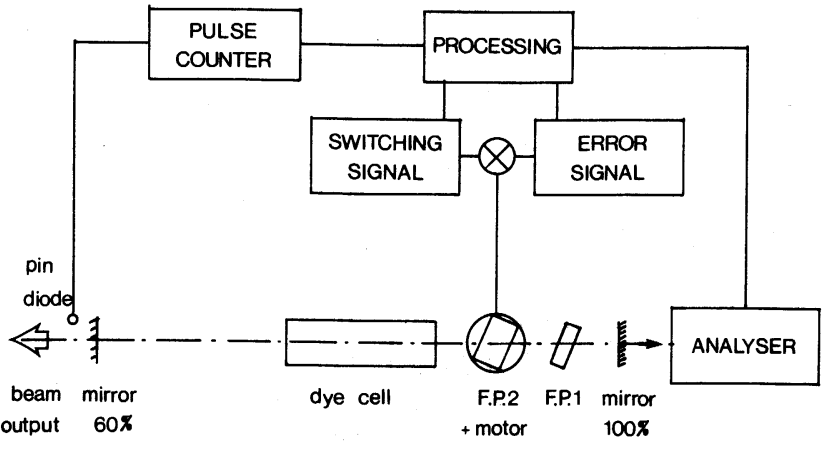

Fig. 7. - Schematic diagram of the wavelength control of a F.P tuned dye laser. The flash lamp pumped dye laser has an hexaelliptical head detailed in J. Y. Allain [13]. The stepping rotation of the F.P. 2 makes the wavelength emission stabilize close to a reference or switch between the two ones.

experiment, the laser linewidth and line position are monitored on a shot to shot basis to improve the data accuracy.

The characteristics of these systems are summarized in table I which includes also the stabilization and switching requirements already performed.

7. Conclusion. - We have designed and constructed a reliable system to stabilize and control the emission wavelength of pulsed dye lasers (nanosecond or microsecond pulse duration) with a resolving power up to $4 \times 10^{6}$. Rapid switching between two or more predetermined wavelengths can be performed within a short given interval of time. Due to its original

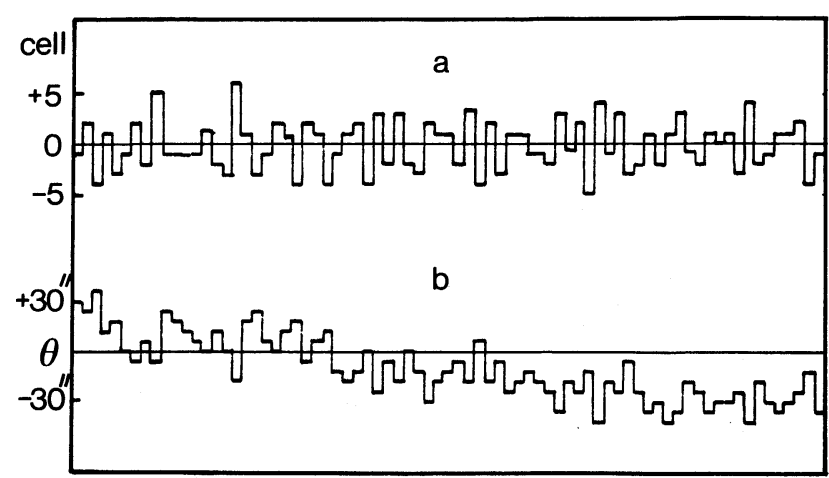

Fig. 8. - a) Temporal fluctuations of the error signal. One cell represents $1.3 \mathrm{~m} \AA . b$ ) Temporal evolution of the position of the tuning element (F.P.2) in the laser cavity. The microsecond pulsed emission is controlled every $0.5 \mathrm{~s} \mathrm{(a} 2 \mathrm{~Hz}$ repetition rate laser) and the angular position of the F.P. 2 is changed by an integer number of steps.

dispersive element, a multibeam Fizeau interferometer, its complete automatization and computer control, our system is of particular interest for spectroscopic applications of pulsed dye lasers and atmospheric laser soundings for both ground based or airborne measurements.

Acknowledgments. - The authors would like to thank C. Leroy and G. Velghe who contributed to the conception and design of this facility and the French Space Agency (C.N.E.S.) which supported the program of development.

\section{References}

[1] Megie, G., Revue Phys. Appl. 14 (1979) 369.

[2] Flamant, P., Megie, G., IEEE J. Quantum Electron. QE-16 (1980) 653.

[3] Pinard, J. and Liberman, S., Opt. Commun. 20 (1977) 344.

[4] Schotland, R. M., Proc. 14th Weather Radar Conference Tucson, Ariz. (1971).

[5] Cahen, C., Megie, G., J. Quant. Spectrosc. Radiat. Transfer. 25 (1981) 151.

[6] SNYDER, J. J., Laser spectroscopy III, Proceedings of the third International Conference, Jackson Lake Lodge, Wyoming, U.S.A., July 4-8, 1977, Ed. J. L. Hall and J. L. Carlsten (Springer Series in Optical Sciences).
[7] Brossel, J., Proc. Phys. Soc. 59 (1947) 224.

[8] Born, M. and Wolf, E., Principles of Optics (Pergamon Press, London), Third edition, 1965.

[9] Reticon Co, G Series Solid State Line Scanners 910 Benicia Ave-Sunnyvale, California 94086.

[10] Talmi, Y., Simpson, R. W., Appl. Opt. 19 (1980) 1401.

[11] Pelon, J., Flamant, P., Chanin, M. L., Megie, G., Proc. Quadriennal International Ozone Symposium, Boulder, Colorado (1980).

[12] Jegou, J. P., Chanin, M. L., Megie, G., Blamont, J. E., Geophys. Res. Lett. 7 (1980) 995.

[13] Allain, J. Y., Appl. Opt. 18 (1979) 287. 